la revue La revue pour l'histoire du CNRS

POUR LHISTOIRE DU CNRS $\quad 16 \mid 2007$

L'expertise scientifique

\title{
Questions de géopolitique ou la géopolitique en question
}

Le cas de l'école française « lacostienne»

Frédéric Encel

\section{OpenEdition}

\section{Journals}

Édition électronique

URL : https://journals.openedition.org/histoire-cnrs/1573

DOI : 10.4000/histoire-cnrs. 1573

ISSN : 1955-2408

Éditeur

CNRS Éditions

Édition imprimée

Date de publication : 3 avril 2007

ISBN : 978-2-271-06453-0

ISSN : 1298-9800

Référence électronique

Frédéric Encel, «Questions de géopolitique ou la géopolitique en question », La revue pour l'histoire du CNRS [En ligne], 16 | 2007, mis en ligne le 26 mars 2009, consulté le 20 mai 2021. URL : http://

journals.openedition.org/histoire-cnrs/1573; DOI : https://doi.org/10.4000/histoire-cnrs.1573

Ce document a été généré automatiquement le 20 mai 2021.

Comité pour l'histoire du CNRS 


\title{
Questions de géopolitique ou la géopolitique en question
}

\author{
Le cas de l'école française « lacostienne»
}

\section{Frédéric Encel}

1 Dans l'absolu, l'absence de reconnaissance de la géopolitique - ou son confinement dans une "case géographie » comme matière en soi -, pourrait prêter à débat; or l'inexistence incompréhensible d'une discipline "relations internationales» le rend caduc et, face à nos principaux amis européens et américains, nous autres Français apparaissons à cet égard fortement lacunaires. De fait, ce qu'on admettra ici comme une démarche intellectuelle et analytique a beau jeu - dans différents instituts de recherche publics et au sein de multiples cercles de réflexion privés - de prétendre combler le vide béant laissé en France en matière d'affaires internationales.

Preuve en est que, boudée voire rejetée des décennies durant, du fait de son utilisation par l'impérialisme des $\mathrm{II}^{\mathrm{e}}$ et $\mathrm{III}^{\mathrm{e}}$ Reich allemands, la géopolitique est devenue à la mode et tout un chacun en fait depuis les années 1990 comme Monsieur Jourdain faisait de la prose. Or, de Jacques Ancel ${ }^{1}$ à Yves Lacoste, non seulement il existe bel et bien une tradition géopolitique française qui, a contrario de l'ancienne géopolitique allemande d'un Ratzel ou d'un Haushaufer, ne s'est jamais inscrite dans une logique pro domo de type nationaliste et/ou impérialiste, mais encore celle-ci répond à des critères de réflexion et une grille d'analyse extrêmement rigoureux et efficients.

Une grille d'analyse contemporaine

Depuis la fondation par le géographe Yves Lacoste, en 1976, de la revue Hérodote, et surtout la création d'une formation doctorale géopolitique en 1989 (à l'université Paris VIII), la démarche intellectuelle géopolitique s'est étoffée pour incarner à présent un véritable "tamis" à travers lequel nombre de conflits sont étudiés, tant par des étudiants et des universitaires que par des journalistes ou de simples citoyens ${ }^{2}$.

Les rivalités de pouvoir

4 Une analyse géopolitique porte avant tout sur des rivalités de pouvoir entre entités politiques, le plus souvent de nature étatique, et visant pour l'essentiel la conquête ou le renforcement de la souveraineté ${ }^{3}$. Cette matière politique précieuse se traduit par 
un contrôle exclusif et simultané par un pouvoir central, d'une part, de symboles de souveraineté (couleurs), d'autre part, d'un système judiciaire - fût-il inique ou indigent - enfin et surtout d'une force armée conséquente.

5 Sur ce dernier élément, notons que si certains États ont renoncé à la possession d'une telle force, c'est pour mieux la déléguer à une puissance tierce et alliée (par exemple l'Islande aux États-Unis, la Mongolie à la Russie, la République centrafricaine à la France, etc.). En revanche, rarissimes sont les États souverains à renoncer officiellement et délibérément à une force armée et à son usage, qu'au demeurant le droit international leur reconnait ${ }^{4}$.

La souveraineté étatique représente un mètre étalon tout à fait incontournable dans l'étude des relations internationales. De fait, nulle terre émergée - si minime et dépourvue d'attraits commerciaux ou stratégiques soit-elle -, n'échappe en principe à un contrôle étatique, et il en va de même pour une part des eaux (zones d'exploitation exclusives, ZEE) et des cieux (espaces aériens). La juste prise en considération de cette recherche effrénée de la souveraineté est, sinon une spécificité de l'analyse géopolitique, du moins l'une de ses particularités fortes; sans cela, on s'expose à de lénifiants lieux communs du type de ceux liés à Jérusalem, « la ville de la paix aimée du même Dieu » (comprendre pourquoi les croyants s'y battent-ils donc?), ou la guerre de façon générale entre ces "hommes qui se ressemblent tant", lesquels poncifs entravent une réelle compréhension des mécanismes menant aux conflits.

Une géographie active

7 Il n'est pas anodin que l'école géopolitique française soit si attachée à la prise en considération prioritaire de la géographie dans ses analyses. Paul Vidal de la Blache et Élisée Reclus ${ }^{5}$ étaient géographes. Or, jusqu'aux années 1970-80, les géographes français s'arc-boutent sur une étude sur-objective de leur discipline, refusant dans leur immense majorité de la voir mêlée aux enjeux et rivalités de pouvoir.

À cet égard, la parution de l'incisif et iconoclaste ouvrage d'Yves Lacoste, La Géographie, ça sert d'abord à faire la guerre ${ }^{6}$, provoqua un véritable scandale au sein de la corporation! Cette prise en compte est d'autant plus stimulante qu'elle tranche non seulement avec le discours ambiant des années 1970-80 selon lequel les capacités balistiques intercontinentales des grandes puissances annulaient de fait toute importance de la géographie, mais encore avec celui des années 1990 d'après la chute du mur de Berlin sur la "fin de l'histoire » et autre "fin des territoires " ${ }^{7} .$. Même voire surtout - sur des territoires exigus, c'est-à-dire sur des niveaux d'analyse de la dizaine de kilomètres, du kilomètre ou de l'hectomètre (cf. le cas de la Vieille ville de Jérusalem) ${ }^{8}$, la géographie demeure prédominante, en particulier pour ce qu'elle révèle et par ce qu'elle contient d'initiatives et de rapports de force tactiques et stratégiques. Sur un micro-territoire où s'opposent des pouvoirs soit dépourvus d'armes de destruction massive, soit dépourvus de la volonté morale et/ou de la capacité politique de s'en servir, on en revient aux fondamentaux tactiques de l'Antiquité : la possession physique d'une simple colline qui favorise alors l'angle de tir (à l'arme à feu individuelle) et le champ de vision (à l'œil nu) offre un avantage certain indépendamment de tout autre considération. En outre, une topographie avantageuse consiste aussi à pouvoir disposer ses couleurs en un point plus élevé que celles de l'adversaire. Pour rester au Proche-Orient, rappelons que le 5 juin 1967, l'aviation israélienne choisit de frapper les aérodromes égyptiens au moment très précis du lever du soleil, particulièrement aveuglant dans le désert cristallin du Sinaï. 
Représentations d'un espace

9 La représentation reste sans doute le concept le plus original, l'outil le plus efficient dans la démarche lacostienne. Il s'agit de la manière dont une population perçoit son histoire collective, son essence, son territoire, ou celles et celui de populations admises comme différentes, en son sein ou à l'extérieur d'un espace donné.

10 Sur un espace conflictuel ou simplement frontalier, la toponymie révèle le plus souvent des caractéristiques géopolitiques passionnantes.

11 Que la Constituante décide, en 1790, de baptiser presque tous les départements français - nouveaux espaces administratifs strictement délimités et destinés à incarner les courroies de transmission du pouvoir républicain -, selon des critères exclusivement géographiques, relève d'une attitude géopolitique : il s'agit de déconstruire le pays de l'Ancien Régime, ce terroir qui évoquait des identités locales fortes et enracinées pouvant servir de futurs leviers séparatistes et/ou sécessionnistes. Ainsi édulcorera-ton l'épaisseur culturelle, politique et linguistique des espaces basques, bretons, ou encore alsaciens en ne mentionnant que des massifs montagneux, des cours d'eau ou des côtes.

12 Au Proche-Orient, l'espace hautement conflictuel baptisé par les Anglo-Saxons « West Bank » (rive occidentale) et par les francophones "Cisjordanie » (du latin, en deçà du Jourdain), n'est jamais désigné comme tel par les deux antagonistes y revendiquant tout ou partie de la souveraineté : ainsi les Israéliens, de gauche comme de droite, laïcs ou sionistes-religieux et dès l'école via les livres scolaires, l'appellent majoritairement "Yehouda veShomron" (Judée et Samarie) ${ }^{9}$. Il va de soi que ce toponyme hébraïque renvoie à un corpus infiniment moins neutre que le fleuve Jourdain, en l'espèce à un territoire biblique détenu par les royaumes hébreux davidiques. Quoi de plus juif que Yehouda, la Judée, dans un pays où Juif se dit yehoudi ?...

Dans le même registre mais sur un continent et dans un conflit différent, on notera que les Serbes admettent le Kosovo comme le berceau de leur patrie, notamment parce que la bataille dite du Champ des merles, menée (et perdue) en 1389 face aux forces ottomanes, s'y déroula ${ }^{10}$. Ajoutons que les aspects démographiques ne pèsent guère dans ce type de représentations ; le Kosovo de 2006 est peuplé à $90 \%$ de non Serbes ${ }^{11}$ et la Cisjordanie/Judée-Samarie n'abritait strictement aucun Juif en 1967, et seulement 250000 en 2006 face à $85 \%$ d'Arabes palestiniens ${ }^{12}$. On pourrait multiplier à l'infini les exemples de lieux et d'espaces dont la dénomination par des puissances sert à légitimer leurs revendications de souveraineté.

Représentations de Soi, de l'Autre

Outre ces perceptions spatiales, il convient d'observer les représentations de sa propre identité, ou de celle d'adversaires intérieurs ou extérieurs. Tantôt elles relèvent d'une dimension victimaire (complexe israélien d'Auschwitz) ou complotiste (thème des croisades dans les sociétés arabes moyen-orientales) poussée à l'extrême, tantôt elles expriment la notion de bastion ou d'îlot face à/dans un monde hostile et majoritaire.

Rien n'est moins anodin que les représentations croisées et souvent mimétiques des Juifs israéliens et des Arabes palestiniens: les premiers se représentent les seconds comme des Arabes avant tout - lesquels pourraient donc parfaitement vivre ailleurs qu'en Palestine, sur ces millions de kilomètres carrés du monde arabe -, les seconds se représentant les Juifs comme des membres d'une religion et non d'un peuple - ce qui implique une ségrégation religieuse et une illégitimité ontologique de l'État d'Israël. 
Bien en amont de tout autre contentieux frontalier, hydrique ou diplomatique, ce fossé abyssal de perception identitaire de l'Autre grève pour l'heure toute chance de paix durable dans la région.

Modes de réduction scientifiques et critiques morales de la géopolitique

$17 \mathrm{Au}$ sein de l'Université, la démarche géopolitique fait régulièrement l'objet d'une double et redoutable accusation; d'une part, elle se fonderait sur un déterminisme de mauvais aloi, et, d'autre part, elle se nourrirait d'un cynisme systématique. Une représentation géopolitique ne se détecte pas à la lumière d'une enquête d'opinion sur un état de fait donné ou un événement ponctuel. Une observation sérieuse des représentations exige deux conditions: elle doit porter sur des «temps longs» braudéliens, et sur des thématiques identitaires.

Qu'un sondage indique que $65 \%$ de personnes interrogées (sur un échantillon dit représentatif de 900 personnes en moyenne) admettent les voitures allemandes comme plus solides que les voitures italiennes ne traduit pas l'existence d'une représentation géopolitique. Le problème ne réside pas seulement dans le caractère hautement photographique de la question; il ne s'agit pas là de perception de type identitaire. Dans une démarche géopolitique, la représentation porte nécessairement - on l'a vu -, sur des éléments politiques faisant sens pour une collectivité, au point parfois d'envisager des sacrifices considérables pour la paix ou la guerre.

L'accusation de déterminisme, ou comment échapper au " café du commerce »

Plusieurs outils très précis constituent autant de critères pour jauger de l'existence d'authentiques représentations géopolitiques : manifestations récurrentes et séculaires d'attachement à un territoire et/ou d'hostilité vis-à-vis d'une population donnée; comptines pour enfants, littérature et chansons populaires; livres d'histoire et de géographie ou d'instruction civique; slogans ou discours officiels répétés; emblèmes nationaux (tribaux, religieux); rejet de l'exogamie avec certains groupes humains, etc. En puisant dans ces éléments de vie politique collective, le géopolitologue rigoureux refuse d'entrer au « café du commerce » évoqué avec mépris par certains contempteurs de la démarche géopolitique, ainsi que dans une logique déterministe puisque les mentalités évoluent bien entendu au fil du temps, notamment en fonction des contextes sociopolitiques et militaires. Il demeure que certaines représentations transcendent des évolutions pourtant profondes et y survivent, par exemple pour ce qui concerne l'antisémitisme au sein de certaines sociétés, les clivages culturels et religieux au Moyen-Orient, ou encore la tribu ancestrale primant l'idée de nation en Afrique noire.

La géopolitique, un machiavélisme?

Le géopolitologue est-il forcément cynique dans le cadre de sa réflexion ou, pire, intrinsèquement? La prise en compte de certaines réalités inhérentes aux relations internationales - primauté (jusqu'à un certain seuil selon les régimes et les personnalités des dirigeants en place) des intérêts économiques ou stratégiques sur les considérations humaines; recours récurrent à l'usage de la force armée; instrumentalisation de thématiques populaires à des fins expansionnistes, etc. Procèdet-elle d'une attitude machiavélique ? En outre, penser la guerre signifie-t-il l'espérer? Certes pas, et Raymond Aron en son temps eut pour grand mérite de réhabiliter non pas le désir de guerre de Clausewitz mais son cadre conceptuel ${ }^{13}$.

21 Mais cette défiance ne proviendrait-elle pas d'une confusion, celle de croire que le géopolitologue souscrit - par faiblesse, facilité, ou empathie pour son sujet d'étude - à 
des représentations jugées belliqueuses, ou, plus grave encore, qu'il excuse des comportements criminels? À cet égard faudrait-il rappeler que comprendre puis expliquer ne signifie pas nécessairement adhérer puis véhiculer, et que l'honnêteté intellectuelle constitue pour le chercheur de bonne foi un garde-fou en principe salvateur? Il ne s'agit pas d'observer une neutralité de type relativiste qui place sur le même piédestal bourreaux et victimes - en cas de crime contre l'humanité notamment ${ }^{14}$-, sous prétexte de rigueur scientifique, mais bien de tendre à l'objectivité, autrement dit à la tentative consistant sans (trop d') a priori à décrypter les représentations antagonistes avec une identique rigueur.

Pour conclure, inversons le postulat: et si une démarche géopolitique rigoureuse, menée sur un conflit armé potentiel ou déjà entamé, permettait de contribuer à l'éviter ou à en atténuer les effets meurtriers ? Une telle espérance ne peut paraître incongrue ou présomptueuse qu'à ceux qui s'arc-boutent sur une conception négative de la géopolitique.

Car à y regarder de près, les conflits excessivement meurtriers et hautement déstabilisateurs des Balkans et des Grands Lacs - pour ne prendre que les années 1990 -, auraient vraisemblablement pu connaître une tournure moins cataclysmique si les décideurs occidentaux, diplomates et hommes d'État, avaient consacré davantage d'efforts à appréhender les mécanismes belligènes et criminels qui se mettaient rapidement en place dans ces deux régions, à commencer par l'instrumentalisation par des pouvoirs politiques autoritaires de représentations populaires. Les nationalismes serbe et croate puisant dans les temps médiévaux et la seconde guerre mondiale d'une part, la construction raciale et fantasmatique hutue à l'encontre des "arrogants nilotiques » tutsis d'autre part, un contexte géographique humain particulier dans les deux cas. Tout cela ne pouvait s'ignorer, au moins chez les experts des Nations Unies sur le maintien de la paix.

Car ne péchons pas par naïveté : dans les cas balkanique et rwandais comme dans presque tous les autres cas de guerre et/ou de crimes contre l'humanité, la vigilance d'analystes compétents pèse peu face aux intérêts réels ou supposés des puissances et des intérêts industriels. Aussi l'on en revient à la sempiternelle realpolitik ne souffrant guère d'ingérence dans le jeu des rapports de force, realpolitik précisément susceptible paradoxe ? - d'être tempérée par... une vraie réflexion géopolitique.

Depuis sa naissance à la fin du XIX ${ }^{\mathrm{e}}$ siècle, la géopolitique contemporaine a subi des évolutions considérables et peut s'étudier sous l'angle épistémologique. Les grands courants et écoles de pensée ;

* en Allemagne, die Geopolitik, repose sur les approches théoriques de Friedrich Ratzel (école de Berlin);

* aux États-Unis, le journaliste et professeur de science politique Nicholas Spykman et l'Amiral Alfred Mahan se sont intéressés aux relations entre le développement technologique des civilisations et la domination de l'espace par les États ;

* en Grande-Bretagne, la sea power définit la puissance anglaise par la domination des mers/océans (théorie de l'empire maritime). 


\section{NOTES}

1.Spécialiste des Balkans puis des frontières européennes de manière générale, Jacques Ancel, quelque peu oublié ou méconnu en France, rédige dans les années 1920-30 une série d'ouvrages à caractère géopolitique, dont Géographie des frontières (Préface d'André Siegfried), Gallimard, 1936, et Géopolitique, Delagrave, 1936. Ancel dispensera également à cette époque le tout premier cours dit de géopolitique, en l'occurrence à la Fondation Andrew Carnegie.

2.Sur les outils et la méthode, on pourra se référer au préambule du Dictionnaire de géopolitique, Y. Lacoste (dir.), Flammarion, 1994.

3.Il convient de noter que depuis plusieurs années, la géopolitique interne connaît un essor certain sous l'impulsion du professeur Béatrice Giblin-Delvallet, directrice de l'Institut français de géopolitique (IFG, anciennement Centre de recherches et d'analyses géopolitiques), de l'université Paris VIII. Il s'agit d'observer des rapports certes conflictuels, mais qui ne débouchent que rarement sur des conflits sanglants et qui éclatent entre entités politiques infra étatiques, telles que des régions, des communes, etc.

4.Notamment par le truchement de l'article 51 de la Charte des Nations Unies consacré à la légitime défense.

5.Paul Vidal de la Blache a fait de la géographie une discipline indépendante en France. Il est à l'origine de l'idée de possibilisme : «La nature propose, l'homme dispose ». Élisée Reclus fut géographe, militant et penseur de l'anarchisme de la fin du XIX ${ }^{\mathrm{e}}$ siècle. 6.Paru aux éditions Maspéro en 1976. L'hostilité des géographes s'estompera progressivement par la suite. Signe des temps : en 2000, un jury du festival géographique de Saint-Dié des Vosges décernera à Yves Lacoste le prix Vautrin Lud pour son œuvre.

7.La Fin de l'Histoire, du titre éponyme de l'ouvrage controversé de l'Américain Francis Fukuyama. Dans un autre registre, le politologue Bertrand Badie, professeur à l'Institut d'études politiques de Paris, publiait La Fin des territoires (Fayard, 1995), puis Un Monde sans souveraineté (Fayard, 1999). On relèvera que depuis la chute du mur de Berlin, il n'y eut jamais autant de créations d'États nations, tandis que le modèle de l'Union européenne semble marquer le pas...

8.Nous prenons ici le parti de circonscrire les exemples censés illustrer notre démonstration à trois zones conflictuelles : Proche-Orient, Balkans et Afrique des Grands Lacs.

9.Pour leur part, les Palestiniens le désignent comme la partie centrale de Filastin, la Palestine.

10.Sondages, articles de presse, discours gouvernementaux et manifestations de rues démontrèrent amplement la persistance de cette représentation, ainsi qu'un vote bien plus récent et effectué longtemps après la chute du pouvoir nationaliste du président Milosevitch ; en octobre 2006, les Serbes adoptèrent en effet une nouvelle constitution dont l'un des points d'orgue était le rappel de l'appartenance historique de la « province » du Kosovo à la Serbie.

11.En l'occurrence des albanophones musulmans, les Serbes étant orthodoxes, représentés comme plus ou moins allogènes. 
12.On pourrait pousser plus loin encore cette logique : avant la première vague d'immigrants sionistes européens de 1881 en Eretz Israël (terre d'Israël), l'ensemble de la lointaine Palestine ottomane censée devenir un foyer national juif ne compte qu'environ 10 \% de Juifs, pas même sionistes eux-mêmes ! Voir Géopolitique d'Israël, Frédéric Encel et François Thual, Seuil, 2004.

13.Lire, de Raymond Aron, Sur Clausewitz, (Préface de Pierre Hassner), Complexe, 2005. 14.Dans le cas du dernier grand génocide en date, celui rwandais des Tutsis perpétré par les Hutus en 1994, on a pu constater ce type de relativisation jusqu'au plus haut niveau du pouvoir. Ainsi durant l'été 1994, le président en exercice de la République française, François Mitterrand affirmait : « Dans ces pays-là, un génocide, c'est pas trop important »... (cit. in Gérard Prunier, Rwanda, le génocide, Dagorno, 1999 - Préface de Patrick de Saint-Exupéry, VII).

\section{RÉSUMÉS}

La géopolitique n'est pas une science. En France, elle n'est même pas répertoriée au CNRS ou dans les commissions de spécialistes, et doit, depuis seulement quelques années, se contenter de figurer au registre " géographie » dans la spécialité " géographie géopolitique ». Frédéric Encel revient sur une discipline en quête de "légitimité » auprès des instances universitaires et de recherche.

Geopolitics is not a science. In France, it doesn't really exist nor in CNRS neither in research committees. Since only a few years, it had been registered as political geography. Frédéric Encel brings up again about a discipline which is seeking its legitimacy closer to university and research authorities.

\section{INDEX}

Mots-clés : politique, pouvoir, géopolitique, géopolitologue, Yves Lacoste, Elisée Reclus, Paul Vidal de la Blache, sea power, Geopolitk, Hérodote, souveraineté, géographie, guerre, micro territoire, espace, territoire, représentation

\section{AUTEUR}

\section{FRÉDÉRIC ENCEL}

Frédéric Encel, politologue, docteur en géopolitique, est professeur de relations internationales à l'ESG, et maître de séminaire à la préparation ENA de l'Institut d'études politiques de Rennes. Il a publié plusieurs ouvrages consacrés au MoyenOrient et à la géopolitique (Géopolitique du sionisme, Armand Colin, 2006). 